\title{
Degradation Analysis on Electrospun Membrane of Polyurethane Blended Chitosan for Cardiovascular Graft
}

\section{Thiviya Selvaras ${ }^{1}$, Saravana Kumar Jaganathan ${ }^{2}$, Sivakumar Sivalingam ${ }^{3}$, Syafiqah Saidin ${ }^{1,4^{*}}$}

\author{
${ }^{1}$ School of Biomedical Engineering \& Health Sciences, Faculty of Engineering, Universiti Teknologi Malaysia, \\ 81310 UTM Johor Bahru, Johor, Malaysia \\ ${ }^{2}$ Department of Engineering, Faculty of Science and Engineering, University of Hull, Hu67RX, United Kingdom \\ ${ }^{3}$ Dept. of Cardiothoracic Surgery, Institut Jantung Negara, 145, Jalan Tun Razak, 50400 Kuala Lumpur, Malaysia \\ ${ }^{4}$ IJN-UTM Cardiovascular Engineering Centre, Institute of Human Centered Engineering, Universiti Teknologi \\ Malaysia, 81310 UTM Johor Bahru, Johor, Malaysia
}

*Corresponding Author syafiqahsaidin@biomedical.utm.my

Received 20 October 2021; Accepted 6 December 2021; Available online 31 Januari 2022

https://doi.org/10.11113/humentech/v1n1.8

\begin{abstract}
:
Coronary heart disease (CHD) is classified as the heart abnormality aside from myocardial damage which results in the blockage of coronary arteries which supply blood to the heart. In this study, a degradable vascular graft was developed to overcome the complications that associated with CHD. The degradable graft was fabricated in a structure of electrospun membranes using polyurethane (PU) matrix blended with chitosan (CS). The degradation analyses were performed through an immersion procedure by conducting the weight loss measurement and the element release test. The blending of PU with CS resulted in the increment of membrane degradability. The CS elements were released from the PU/CS membranes following the Higuchi model release mechanism. As a conclusion, the PU/CS membranes possess the degradable properties which might serve as a plausible candidate for the development of vascular graft for repairing cardiac damage.
\end{abstract}

Keywords: Electrospinning; Polyurethane; Chitosan; Membrane; Degradation

\section{Introduction}

Cardiovascular diseases (CVD) are one of the dominant cases which cause millions of deaths worldwide. Stroke, heart failure, hypertension, congenital heart disease and cardiac infarction are several illnesses associated to CVD [1]. Coronary heart disease (CHD) is another heart abnormality aside from myocardial damage which results in blockage of the coronary arteries which supplies blood to the heart and it accounts almost 50\% deaths associated with CVD. Millions of people are suffering from CHD where it is considered as one of the major health issues in the world [2]. So, there is a demand for artificial cardiovascular graft in order to recover from the CHD [3]. The tissue engineered vascular grafts have gained a huge attention for repairing the coronary heart disease and myocardium damage [4]. The use of synthetic polymer scaffolds in tissue engineering applications for the replacement of injured native vessels has gotten a lot of attention which provides structural and functional support for cell attachment and growth [5]. However, their utilization is limited in biomedical applications due to certain limitations such as inability to remodeling or regenerate native tissues, loss of mechanical strength over time and increased risk of infection [6]. 
Polyurethane is widely used in making tissue engineered scaffolds as the fabrication of nanofibers from PU is easy and also possesses biodegradable, excellent biocompatibility and elastic mechanical properties [7]. In addition, its slow degradation rate can be considered helping in tissue replacement such as blood vessels which demand mechanical strength due to the high risk to the patient associated with a non-optimal balance of scaffold degradation rates and new extracellular matrix production [8]. Even though, this synthetic polymer provides sufficient mechanical strength but they are lack in bio-functionalization due to their hydrophobic nature. Therefore, the combination of synthetic and natural polymers in constructing the scaffold is one of the approaches in addressing the complication of bio-functionalization [9]. Natural polymers provide many advantages such as hydrophilicity, cellular affinity and often degrade which give the spaces for cell migration and proliferation [10]. In this study, chitosan (CS) a natural resource compound derived from chitin, was used to enhance the properties of the developed graft [11]. It has been mainly used in pharmaceutical, biomedical industries, tissue engineering and wound dressing, due to its hydrophilicity and good biodegradability [12].

Both PU and CS were blended together for the fabrication of graft membrane due to their specific enhancement properties. In order to form the membrane, an electrospinning technique was adopted as this technique is unique, versatile and cost effective which applies high voltage to produce polymeric nanofibers which is suitable for vascular graft applications [13]. The fabricated nanofibers through this technique have been reported to possess high porosity which provides large surface area for cell penetration, cell adhesion and development of extracellular matrix [14].

The degradation properties of a biomaterial polymer play an important role on the long-term performance of tissueengineered cell/material construct. Degradation kinetics can affect the cellular responses such as cell growth, tissue regeneration and host response [15]. The correlation between degradation rate and cell activity will lead to tissues formation, known as an important process in tissue regeneration [16]. In the case of bone or cell repairs, the biomaterial graft or scaffold should be degraded over time which giving way to cell regeneration in enabling the complete reconstruction of structure and function of the native tissues [17]. Synthetic graft should possess an optimal degradation rate and should be biodegradable that helps in developing tissue regeneration. However, some behaviour of the graft is limited due to the indifferent degradation rate where it caused destructive changes in structural integrity which influence the overall cell integration process [18]. In this study, PU electrospun membranes were incorporated with CS for the potential use as vascular grafts. The degradation test was conducted on the PU electrospun membranes incorporated with $\mathrm{CS}$, followed by two post-analyses of weight measurement and element release, to forecast the performances of these membranes following an implantation.

\section{Materials and Methods}

\subsection{Sample preparation}

Chitosan (CS), N, N-dimethylformamide (DMF), acetic acid and phosphate buffer saline (PBS) were supplied by Sigma-Aldrich, Germany. Polyurethane (Tecoflex EG-80A) was purchased from Lubrizol, USA.

A homogenous solution of $8 \%(\mathrm{w} / \mathrm{v})$ PU was prepared by dissolving $800 \mathrm{mg}$ of PU with $10 \mathrm{~mL}$ of DMF and stirred for $24 \mathrm{hrs}$ at room temperature [19]. Concurrently, a homogenous solution of 3\% CS solution was prepared by dissolving $300 \mathrm{mg}$ of CS powders in $10 \mathrm{~mL}$ of DMF for $24 \mathrm{hrs}$ at room temperature. Both homogenous solutions were mixed at (7:3) $\mathrm{v} / \mathrm{v} \%$ to form PU/CS composite under vigorous stirring for $1 \mathrm{hr}$.

The electrospun membranes were fabricated using an electrospinning technique. The homogenous solution of pure PU and PU/CS were electrospun at a flow rate of $0.5 \mathrm{~mL} / \mathrm{hr}$ with the applied voltage of $12 \mathrm{kV}$. The nanofibers were deposited on the collector plate which was placed $15 \mathrm{~cm}$ away from the needle for $8 \mathrm{hrs}$. The deposited electrospun membranes were retrieved carefully for further analysis.

\subsection{Weight loss measurement}

The degradation of the electrospun membranes were investigated in-vitro by ageing the membranes $(3 \mathrm{~cm} \times 3 \mathrm{~cm})$ in PBS at pH 7.4, in three replicates [20]. All samples were placed individually in centrifuge tubes containing PBS and kept at temperature of $37^{\circ} \mathrm{C}$. At time periods of 5, 10,20,30,60 days, the membranes were collected, washed thoroughly with distilled water and then oven-dried to measure their weight loss. The weight loss was calculated using Equation 1 [21].

$$
\text { Weight loss }(\%)=\frac{\text { Initial-Final }}{\text { Initial }} \times 100
$$




\subsection{Element release test}

The element release from the electrospun membranes was observed through an element release test using UV-Vis spectrophotometer (GENESYS 10S UV-Vis, Thermo Scientific, US). All samples were placed individually in centrifuge tubes containing deionized water and kept at temperature of $37^{\circ} \mathrm{C}$. The deionized water was refreshed at time points of $5,10,20,30,60$ days [22]. The aged deionized water was collected for the analysis of element release at an absorption wavelength of $290 \mathrm{~nm}$ for the CS measurement. The element release records were plotted based on the standard curve and fitted to four mathematical modellings of zero-order, first-order, Higuchi and Korsmeyer-peppas. The best fitted graph $\left(\mathrm{R}^{2}\right.$ value closest to 1$)$ was acquired by linear slope to illustrate the possible element release mechanism of the electrospun membranes.

\section{Results and Discussion}

\subsection{Weight loss measurement}

The degradation of both electrospun membranes were studied by incubating the membranes in PBS and measuring their weight loss at different time intervals as plotted in Figure 1. A higher mass loss for the PU/CS electrospun membranes was observed at all time points when compared to the PU membranes. The weight loss for both PU and PU/CS membranes were increased as the degradation time increased. The PU electrospun membranes exhibited a weight loss of $2.23 \pm 0.36 \%$ at day $5,2.75 \pm 0.24 \%$ at day $10,2.92 \pm 0.36 \%$ at day $20,3.09 \pm 0.24 \%$ at day 30 and $3.26 \pm 0.36 \%$ at day 60. The PU membranes showed low degradation capability which might be attributed to its low hydrophilicity and consequently low effective collision with water molecules [23]. While, the PU/CS exhibited a weight loss of $2.57 \pm 0.44 \%$ at day $5,3.20 \pm 0.30 \%$ at day $10,3.62 \pm 0.30 \%$ at day $20,3.82 \pm 0.44 \%$ at day 30 and $4.24 \pm 0.44 \%$ at day 60 . The incorporation of CS into the PU matrix has induced the weight loss and accelerated the degradation process. A previous study mentioned that CS is trapped in PU network by weak bonds between hydroxyl (-OH) and amine (-NH²) [24]. Following the incubation in the PBS solution, the bond was cleavage and the CS were removed from the membranes which facilitated the movement of water molecules into the PU network for greater degradation response. The weight loss of the samples confirmed their degradation process after the incubation. The membrane surface of the samples was lightly eroded. Both membrane surface seemed to be smoother than the original sample.

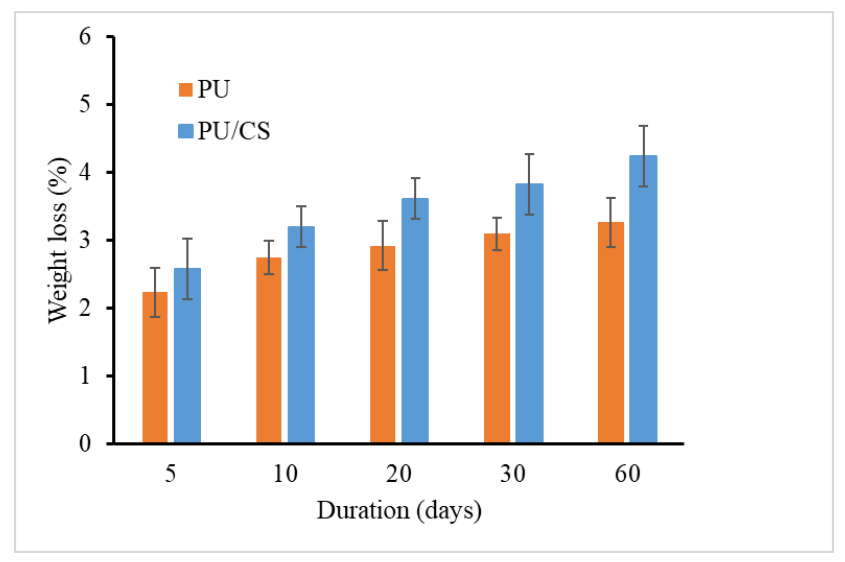

Figure 1. Weight loss measurement of PU and PU/CS at different time intervals (5, 10, 20, 30 and 60 days)

\subsection{Element release test}

The element release of CS was investigated with UV-Vis spectroscopy at an absorption wavelength of $290 \mathrm{~nm}$. The ageing process was carried out using deionized water to determine the release mechanism of CS from the electrospun membranes. The release profile showed an initial burst release for the PU/CS electrospun membrane followed by a stable release of CS after day 5 as shown in Figure 2. Initially, the fast release was observed due to the rapid dissolution of the absorbed element. Then, the diffusion of water molecules into the membranes was reduced due to the entrapment of element within the membranes [25]. There was no element release data recorded on the PU membrane as there was no CS release from the pure PU membranes. 


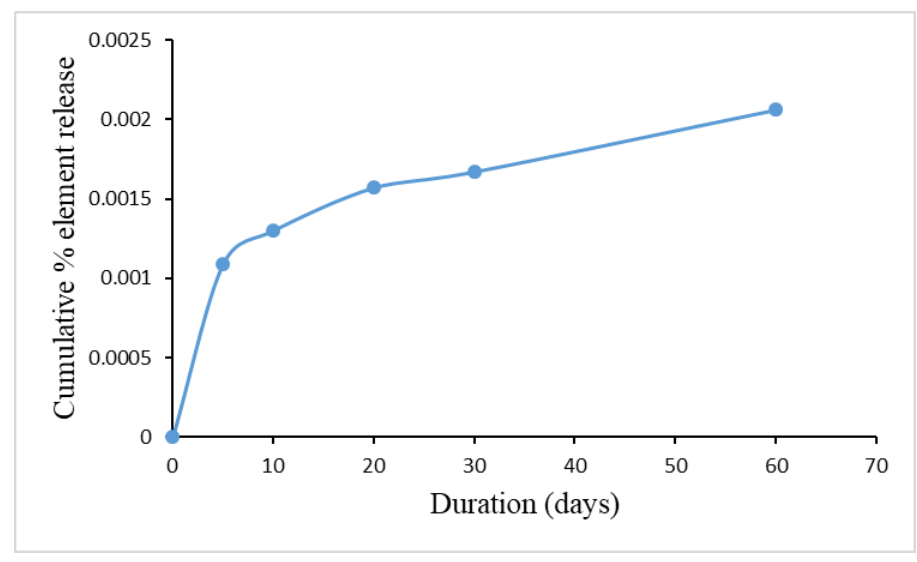

Figure 2. Percentages of cumulative CS release from PU/CS membrane

The zero order, first order, Higuchi kinetic and Korsmeyer Peppas models were used to study the element release mechanism. The mathematical model with the highest $\mathrm{R}^{2}$ was selected as the best model to describe the dissolution data. Table 1 shows the $\mathrm{R}^{2}$ that obtained after fitting various release kinetic models to the CS release data. The release pattern of the PU/CS membranes was fitted into Higuchi model which describes the rate of diffusion from the membrane surface to the solution was in a significant contribution. The initial element concentration in the system is much higher than the solubility of the element provides the basis for the justification of the applied pseudo-steady-state approach in Higuchi [26].

Table 1. Determinant coefficient, $\mathrm{R}^{2}$ of mathematical models for CS release from PU/CS membranes

\begin{tabular}{ccccc}
\hline Sample & Zero Order & First Order & Higuchi & $\begin{array}{c}\text { Korsmeyer } \\
\text { Peppas }\end{array}$ \\
\hline PU/CS & 0.66 & 0.66 & 0.91 & 0.57
\end{tabular}

\section{Conclusion}

The PU electrospun membranes blended with CS were fabricated using an electrospinning technique. The incorporation of CS into the PU membranes increased the weight loss and induced the release of CS following the Higuchi model, which contributed to the increment of degradation properties. Therefore, the PU/CS membranes were considered degradable that might serve as a plausible candidate for the repairing of cardiac damage.

\section{References}

[1] N. Syazana and I. Sukmana, Electrospun-based fibrous scaffold for cardiovascular engineering applications: A review, ARPN Journal of Engineering and Applied Sciences, 2016, 11(7):4778-4781.

[2] D. V. D. Linde, E. E. M. Konings, M. A. Slager, M. Witsenburg, W. A. Helbing, J. J. M Takkenberg and J. W. Roos-hesselink, Birth prevalence of congenital heart disease worldwide a systematic review and meta-analysis, Journal of the American College of Cardiology, 2011, 58(21):2241-2247. https://doi.org/10.1016/j.jacc.2011.08.025

[3] N. M. Pinto, H. T. Keenan, L. L. Minich, M. D. Puchalski, M. Heywood and L. D. Botto, Barriers to prenatal detection of congenital heart disease: A population-based study, Ultrasound in Obstetrics and Gynecology, 2012, 40(4): 418-425. https://doi.org/10.1002/uog.10116

[4] Q. Wang, H. Yang, A. Bai, W. Jiang, X. Li, X. Wang, Y. Mao, F. Guo, T. Ding, H. Chen, S. Chen, J. Zhang, C. Liu and N. Sun, Functional engineered human cardiac patches prepared from nature's platform improve heart function after acute myocardial infarction, Biomaterials, 2016, 105:52-65. https://doi.org/10.1016/j.biomaterials.2016.07.035

[5] M Kitsara, P. Joanne, S. E. Boitard, I. B. Dhiab, B. Poinard, P. Menasché, C. Gagnieu, P. Forest, O. Agbulut, Y. Chen, Microelectronic engineering fabrication of cardiac patch by using electrospun collagen fibers, Microelectronic Engineering, 2015, 144:46-50. https://doi.org/10.1016/j.mee.2015.02.034

[6] T. L. Mirensky and C. K. Breuer, The development of tissue-engineered grafts for reconstructive cardiothoracic surgical applications, Pediatric Research, 2008, 63(5):559-568. https://doi.org/10.1203/01.pdr.0000305938.9 $\underline{2695 . b 9}$ 
[7] A. Silvestri, P. M. Serafini, S. Sartori, P. Ferrando, F. Boccafoschi, S. Milione, L. Conzatti, and G. Ciardelli, Polyurethane-based biomaterials for shape-adjustable cardiovascular devices, Journal of Applied Polymer Science, 2010, 116(5):2658-2667. https://doi.org/10.1002/app/

[8] N. Detta, C. Errico, D. Dinucci, D. Puppi, D. A. Clarke, G. C. Reilly and F. Chiellini, Novel electrospun polyurethane/gelatin composite meshes for vascular grafts, Journal of Materials Science Materials in Medicine, 2010, 21(5):1761-1769. https://doi.org/10.1007/s10856-010-4006-8

[9] K. Garg, S. A. Sell, P. Madurantakam and G. L. Bowlin, Angiogenic potential of human macrophages on electrospun bioresorbable vascular grafts, Biomedical Materials, 2009, 4(3):031001. https://doi.org/10.1088/1748$\underline{6041 / 4 / 3 / 031001}$

[10] J. F. Mano, G. A. Silva, H. S. Azevedo, P. B. Malafaya, R. A. Sousa, S. S. Silva, L. F. Boesel, J. M. Oliveira, T. C. Santos, A. P. Marques, N. M. Neves and R. L. Reis, Natural origin biodegradable systems in tissue engineering and regenerative medicine: Present status and some moving trends, Journal of the Royal Society Interface, 2007, 4(17):999-1030. https://doi.org/10.1098/rsif.2007.0220

[11] E. Y. -H. Lin, A study of the mobility of silver ions in chitosan membranes, UWSpace, 2007. https://uwspace.uwaterloo.ca/handle/10012/3097

[12] S. Aisverya and P. N. Sudha, f- Multiwalled carbon nanotube-grafted-chitosan/polyvinylpyrrolidone blends: Preparation and characterization, Scholars Research Library, 2014, 6(3):9-14.

[13] A. Cooper, N. Bhattarai and M. Zhang, Fabrication and cellular compatibility of aligned chitosan-PCL fibers for nerve tissue regeneration, Carbohydrate Polymer, 2011, 85(1):149-156. https://doi.org/10.1016/j.carbpol.2011.0 $\underline{2.008}$

[14] J. Kucinska-Lipka, I. Gubanska, H. Janik and M. Sienkiewicz, Fabrication of polyurethane and polyurethane based composite fibres by the electrospinning technique for soft tissue engineering of cardiovascular system, Materials Science and Engineering C, 2015, 46:166-176. https://doi.org/10.1016/j.msec.2014.10.027

[15] C, Grasl, H. Bergmeister, M. Stoiber, H. Schima and G. Weigel, Electrospun polyurethane vascular grafts: In vitro mechanical behaviour and endothelial adhesion molecule expression, Journal of Biomedical Materials Research Part A, 2009, 93(2): 716-723. https://doi.org/10.1002/jbm.a.32584

[16] D. Ren, H. Yi, W. Wang and X. Ma, The enzymatic degradation and swelling properties of chitosan matrices with different degrees of N -acetylation, Carbohydrate Research, 2005, 340(15):2403-2410. https://doi.org/10.1016/j.carres.2005.07.022

[17] L.C. Abraham, J. F. Dice, R. F. Finn, N. T. Mesires, K. Lee and D. L. Kaplan, Extracellular matrix remodelingMethods to quantify cell-matrix interactions, Biomaterials, 2007, 28(2):151-161. https://doi.org/10.1016/j.biomaterials.2006.07.001

[18] S. H. Park, E. S. Gil, H. Shi, H. J. Kim, K. Lee and D. L. Kaplan, Relationships between degradability of silk scaffolds and osteogenesis, Biomaterials, 2010, 31(24):6162-6172. https://doi.org/10.1016/j.biomaterials.2010.04.028

[19] R. Subramaniam, M.P. Mani and S. K. Jaganathan, Fabrication and testing of electrospun polyurethane blended with chitosan nanoparticles for vascular graft applications, Cardiovascular Engineering and Technology, 2018, 9(3):503-513. https://doi.org/10.1007/s13239-018-0357-y

[20] J. Brzeska, M, Morawska, A. Heimowska, W. Sikorska and A. Tercjak, Degradability of cross-linked polyurethanes/ chitosan composites, Journal on Chemistry, Technology and Polymer Processing, 2017, 62(7-8):567-575. https://doi.org/10.14314/polimery.2017.567

[21] H. Hajiali, J. A. Heredia-Guerrero, I. Liakos, A. Athanassiou and E. Mele, Alginate nanofibrous mats with adjustable degradation rate for regenerative medicine, Biomacromolecules, 2015, 16(3):936-943. https://doi.org/10.1021/bm501834m

[22] N. M. Daud, N. A. N. N. Malek and S. Saidin, Influence of coating time on immobilization of chlorhexidine on polydopamine influence of coating time on immobilization of chlorhexidine on polydopamine grafted stainless steel 316L: Characterization, adsorption and cytotoxicity analyses, Proceedings of the 2017 International Conference on Computational Biology and Bioinformatics, 2017, 97-100. https://doi.org/10.1145/3155077.3155095

[23] N. Baheiraei, H. Yeganeh, J. Ai, R. Gharibi, M. Azami and F. Faghihi, Synthesis, characterization and antioxidant activity of a novel electroactive and biodegradable polyurethane for cardiac tissue engineering application, Materials Science and Engineering C, 2014, 44:24-37. https://doi.org/10.1016/j.msec.2014.07.061

[24] D. Y. Zuo, Y. Z. Tao, Y. B. Chen and W.L. Xu, Preparation and characterization of blend membranes of polyurethane and superfine chitosan powder, Polymer Bulletin, 2009, 62(5):713-725. https://doi.org/10.1007/s00289-009-0049-8

[25] N. Islam, I. Dmour and M. O. Taha, M. O, Degradability of chitosan micro/nanoparticles for pulmonary drug delivery, Heliyon, 2019, 5(5):e01684. https://doi.org/10.1016/j.heliyon.2019.e01684

[26] S. S. Dukhin and M. E. Labib, Theory of effective drug release from medical implants based on the Higuchi model and physico-chemical hydrodynamics, Colloids and Surfaces A Physicochemical and Engineering Aspects, 2012, 23(1):1-7. https://doi.org/10.1016/j.colsurfa.2012.04.040 